\title{
Performance of DS-CDMA Cellular Forward Channel
}

\author{
Jan E. Tighe, Member, IEEE, and Tri T. Ha, Fellow, IEEE \\ Department of Electrical Engineering \\ Naval Postgraduate School, Monterey CA 93943-5000
}

\begin{abstract}
We develop the forward channel model for a DSCDMA cellular system operating in a slow-flat Rayleigh-fading and lognormal-shadowing environment. Forward error correction is integrated into the analysis by applying convolution encoding with soft-decision decoding. The worstcase probability of bit error for a mobile user at the edge of the center cell of a seven-cell cluster is developed using Gaussian approximation. We also incorporate user distribution and power control and antenna sectoring into our analysis.
\end{abstract}

\section{INTRODUCTION}

The performance of the forward channel of a DS-CDMA cellular system becomes increasingly more important as we transition from a system that carries principally voice traffic to one that supports data traffic at high data rates. The forward signal traveling through the mobile radio channel is subject to reflection, diffraction, and scattering. Rayleigh fading channels are typically used to model the small-scale propagation effects on the forward signal [1]. Large-scale propagation models incorporate shadowing effects on path loss using a lognormal random variable [2]. Furthermore, the key-limiting factor on DS-CDMA performance is the cochannel interference. Convolutional encoding with softdecision decoding, antenna sectoring, and power control will be employed to overcome interference in $3 \mathrm{G}$ systems [3]. Investigations into the performance of the cellular DSCDMA forward channel [4]-[5] have considered a subset of these channel conditions and/or performance enhancing techniques. Similarly, analysis of the reverse channel [6] [8] has not considered the combined effects of the channel in conjunction with forward error correction and soft-decision decoding. In this paper we use detailed analysis of the forward channel in a Rayleigh-fading and lognormalshadowing environment to develop the upper bound on the probability of bit error for a DS-CDMA cellular system employing convolutional encoding with soft-decision decoding, antenna sectoring, and power control.

\section{CHANNEL MODEL}

In this paper, the DS-CDMA cellular forward channel is modeled as undergoing slow-flat Rayleigh fading and lognormal shadowing. We model the cells in our CDMA system as hexagons using a basic seven-cell cluster with a base station located in the center of each cell. The forward signal $S_{i}(t)$ is transmitted by base station in each cell. It includes the traffic for all active channels in the cell. The power spectrum of this traffic has been spread by a factor of
$N$ and then BPSK modulated. The composite signal $\mathrm{S}_{0}(t)$ transmitted from the base station in the center cell is given by

$$
\mathrm{S}_{0}(t)=\sum_{k=0}^{K-1} \sqrt{2 \mathrm{P}_{\mathrm{t}, k}} b_{k}(t) \mathrm{w}_{k}(t) c(t) \cos \left(2 \pi f_{c} t\right) \text {. }
$$

The transmit power in user channel $k$ is given by $\mathrm{P}_{\mathrm{t}, k}$ and there are $K$ active channels in the center cell. The information signal consisting of a binary bit stream for the $k$ th user is represented as $b_{k}(t)$. The orthogonal Walsh function for the $k$ th user channel in the center cell is represented as $\mathrm{w}_{k}(t)$. The PN spreading code for the center cell is represented as $c(t)$ and the carrier frequency is $f_{c}$. The forward signal is attenuated as a function of distance to the mobile and affected by slow-flat Rayleigh fading and lognormal shadowing. The total signal received by a mobile user in the center cell is comprised of the forward signal sent from the user's base station, interference from the six adjacent base stations, and Additive White Gaussian Noise (AWGN) $n(t) \sim \mathrm{N}\left(0, \mathrm{~N}_{0} / 2\right)$ defined by

$$
\begin{aligned}
r(t)= & \sum_{k=0}^{K-1} R \sqrt{2 P_{k}} b_{k}(t) \mathrm{w}_{k}(t) c(t) \cos \left(2 \pi f_{c} t\right) \\
& +\sum_{i=1}^{6} \sum_{j=0}^{K_{i}-1} R_{i} \sqrt{2 P_{i j}} b_{i j}\left(t+\tau_{i}\right) \mathrm{w}_{i j}\left(t+\tau_{i}\right) \bullet \\
& c_{i}\left(t+\tau_{i}\right) \cos \left(2 \pi f_{c} t+\varphi_{i}\right)+n(t),
\end{aligned}
$$

where $R$ and $R_{i}$ represent the Rayleigh fading effects on the envelope of the forward signals. The lognormal shadowing is represented in the random variable $P_{k}$, which is defined by

$$
P_{k}=\frac{\mathrm{P}_{\mathrm{t}, k}}{L_{H}(d) X}=\frac{\mathrm{F}_{k} \mathrm{P}_{\mathrm{t}}}{L_{H}(d) X},
$$

where $\mathrm{F}_{k}$ is a power control factor for the $k$ th channel; $\mathrm{P}_{\mathrm{t}}$ is a baseline transmit power; $L_{H}(d)$ is a distance $d$ dependent path loss predicted by the extended Hata model [9]; $X \sim \Lambda\left(0, \lambda \sigma_{d B}\right)$ is the lognormal shadowing random variable, where $\lambda=\ln 10 / 10$ and $\sigma_{d B}$ is the standard deviation of an underlying zero-mean Gaussian random variable (in decibels).

\section{PERFORMANCE OF THE BASIC SYSTEM}

\section{A. Uncoded Probability of Bit Error $\widetilde{\mathrm{P}_{e}}$}

In order to assess the performance on the forward channel, we take mobile user one to be located in a corner of the hexagon. The received signal defined by (2) is coherently 
demodulated and we use a Gaussian approximation for the decision statistic as developed in [10]. Accordingly, the uncoded probability of error is given by

$$
\tilde{\mathrm{P}_{e}}=\int_{-\infty}^{\infty} \int_{0}^{\infty} \mathrm{Q}\left(\sqrt{\frac{r^{2} p_{1}}{\frac{1}{3 N} \sum_{i=1}^{6} \sum_{j=0}^{K_{i}-1} \mathrm{E}\left\{R_{i}^{2}\right\} \mathrm{E}\left\{P_{i j}\right\}+\frac{\mathrm{N}_{0}}{2 T}}}\right) \cdot
$$

where $\mathrm{p}_{R}(r)$ and $\mathrm{p}_{P_{1}}\left(p_{1}\right)$ are the probability distribution functions for the Rayleigh and lognormal random variables, respectively and the Q-function is given by

$$
\mathrm{Q}(x)=\int_{x}^{\infty} \frac{1}{2 \pi} e^{\frac{-y^{2}}{2}} d y .
$$

The performance of the uncoded cellular system under normal operating conditions is quite poor $\left(\widetilde{P_{e}} \approx 1 / 2\right)$. Accordingly, for our performance analysis to be meaningful, we add forward error correction (FEC) coding to the system

\section{B. Coded Probability of Bit Error $\mathrm{P}_{e}$}

In order to improve the performance of the DS-CDMA cellular system operating in the Rayleigh-lognormal channel, we institute forward error correction in the form of convolutional codes. We use an $(n, k)$ encoder and the code rate is given by $\mathrm{R}_{c c}=\mathrm{k} / \mathrm{n}$. The coded bits are spread, orthogonally covered, BPSK modulated, and transmitted by the base stations, in the same manner as the uncoded bits. To decode the information bit stream, we use the Viterbi algorithm with soft-decision decoding. In [10], we analyzed the performance of the coded system in a manner similar to that in [1], in which the Viterbi algorithm was applied to an additive white Gaussian noise channel with soft-decision decoding. The first-event error probability $P_{2}(d)$ is determined using a Gaussian approximation as follows:

$$
\left.\mathrm{P}_{2}(d)\right|_{r_{l}, \tilde{x}_{l}}=\mathrm{Q}\left(\sqrt{\frac{\sum_{l=1}^{d} r_{l}^{2} \tilde{x}_{l}}{\alpha}}\right),
$$

where

$$
\alpha=\frac{\mathrm{E}\{X\}}{3 N} \sum_{i=1}^{6} \sum_{j=0}^{K_{i}-1} \frac{\mathrm{F}_{i j}}{\mathrm{~F}_{1}} \frac{L_{H}(\mathrm{~d})}{L_{H}\left(\mathrm{D}_{i}\right)}+\frac{\mathrm{N}_{0}}{2 E_{c}} .
$$

We take $\mathrm{E}\left\{R_{i}^{2}\right\}=1$, and as developed in [10], $\mathrm{E}\left\{1 / X_{i}\right\}=\mathrm{E}\{X\}$. $\widetilde{X}_{I} \sim \Lambda\left(0, \lambda \sigma_{d B}\right)$ is the original form of the lognormal shadowing variable. The first-event error probability is conditioned on the sum of $d$ multiplicative chi-square(with two degrees of freedom)-lognormal random variables, which we represent as

$$
Z_{d}=\sum_{l=1}^{d} R_{l}^{2} \widetilde{X_{l}} .
$$

In order to remove the conditioning of $\mathrm{P}_{2}(d)$, we must determine the pdf for $Z_{d}$ and integrate across it as follows:

$$
\mathrm{P}_{2}(d)=\int_{-\infty}^{\infty} \mathrm{Q}\left(\sqrt{\frac{z_{d}}{\alpha}}\right) \mathrm{p}_{z_{d}}\left(z_{d}\right) d z_{d},
$$

or simulate the integral in (9) using Monte Carlo methods. In [10], we approximated $Z_{d}$ as a multiplicative chisquare(with $2 d$ degrees of freedom)-lognormal random variable. The details of this approximation are addressed in a separate Globecom 2001 conference paper.

Using the approximation or Monte Carlo simulations, we can determine probability of first-event error for a path that is distance $d$ from the all-zero path and that merges with the all-zero path at node B. For a given convolutional code, there are many such paths of differing distances that could merge with the all-zero path at node B . Accordingly, we can calculate an upper bound on the probability of bit error $P_{e}$ using the total number of information bit errors $\beta_{d}$ that is associated with selecting a path of distance $d$ from the all zero path

$$
\mathrm{P}_{e} \leq \frac{1}{k} \sum_{d=d_{\text {free }}}^{\infty} \beta_{d} \mathrm{P}_{2}(d)
$$

Accordingly, we have developed a probability of error $P_{e}$ for the coded DS-CDMA cellular system in the Rayleighlognormal channel for a mobile user in the worst-case position within the cell. As an example, we examine the performance of the DS-CDMA system using the first five terms of the union bound in (10) for a rate $1 / 2$ convolutional encoder with constraint length $v=8 ; d_{\text {free }}=10$ and $\beta_{10}=2, \beta_{11}=22, \beta_{12}=60, \beta_{13}=148, \beta_{14}=340 . \quad \mathrm{We}$ compare the probability of bit error for AWGN, Rayleigh fading, and Rayleigh-lognormal channels as predicted by our

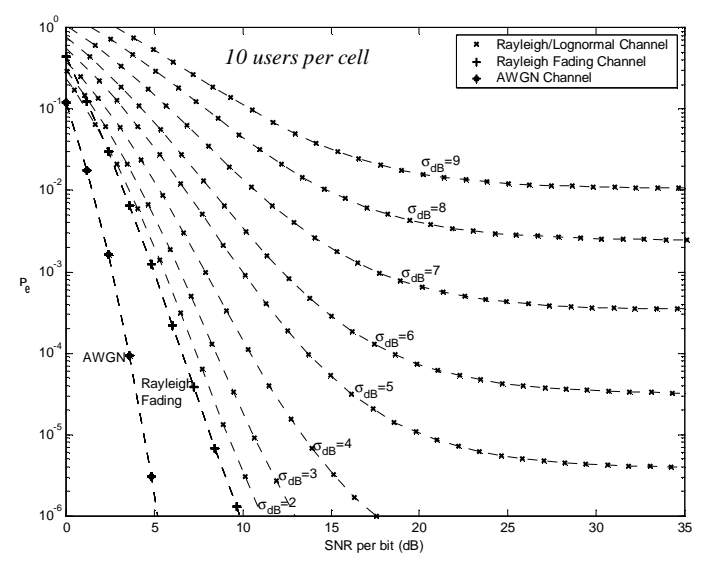

Fig. 1 Probability of Bit Error for DS-CDMA with FEC in Various Channel Conditions with 10 users per cell $\left(\mathrm{R}_{\mathrm{cc}}=1 / 2\right.$ and $\left.v=8\right)$. 
approximation. Fig. 1 shows these results for 10 users per adjacent cell and where we vary the lognormal shadowing parameter $\sigma_{d B}$ from 2 to 9 . We have normalized the cell radius to $\mathrm{d}=1 \mathrm{~km}$ and taken the code spread to be $N=128$. We established the Hata loss for a large city with the base station antenna height at $30 \mathrm{~m}$, the mobile antenna at $1 \mathrm{~m}$, and carrier frequency, $f_{\mathrm{c}}=2000 \mathrm{MHz}$. As depicted in Fig. 1, when lognormal shadowing is added to a pure Rayleigh fading Channel, the probability of bit error drifts away from that of the pure Rayleigh channel as we increase the lognormal shadowing parameter from 2 to 9 . We can also consider performance of the DS-CDMA system with FEC in terms of the number of users per cell that can be supported at a given SNR per bit. If we fix the average SNR per bit at 15 $\mathrm{dB}$, we can predict the probability of bit error for a range of users and for $\sigma_{d B}=2,3, \ldots, 9$ as shown in Fig. 2 .

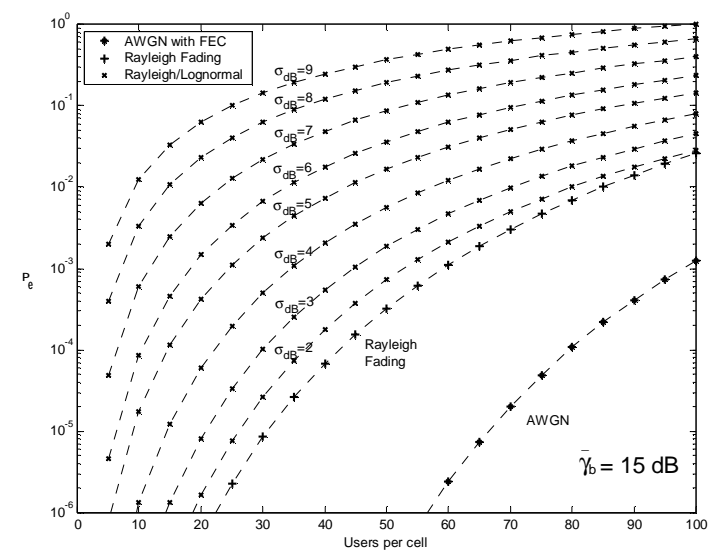

Fig. 2 Probability of Bit Error for DS-CDMA with FEC in Various Channel Conditions with the Average $\mathrm{SNR}=15 \mathrm{~dB}\left(\mathrm{R}_{\mathrm{cc}}=1 / 2\right.$ and $\left.v=8\right)$.

We can further limit the amount of intercell interference by sectoring the cells into 3 or 6 sectors of 120 or 60 degree sectors, respectively by replacing the omni-directional antenna at the base station with several directional antennas. By reducing the intercell interference with sectoring, the performance of our DS-CDMA cellular system operating in a channel with Rayleigh fading and lognormal shadowing is greatly improved. Fig. 3 shows the dramatic increase in performance for the case of $\sigma_{d B}=7$.

\section{Power Control of THE Forward ChanNel}

\section{A. Incorporating User Distribution}

In Section III, we analyzed the performance of the forward channel in a DS-CDMA cellular system by assuming that our intended mobile user was situated in the outer corner of the hexagonal cell. We can obtain a more realistic view of typical performance of the forward channel by assuming that the users are randomly distributed within the cell according to a specified probability distribution. The position of the

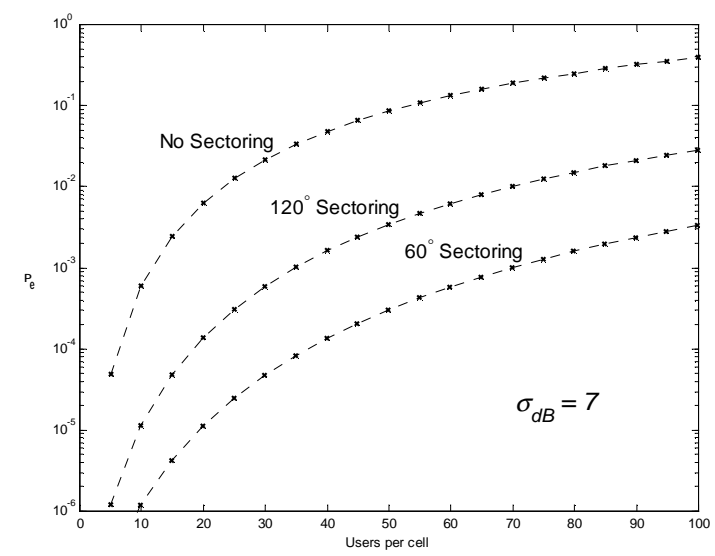

Fig. 3 Probability of Bit Error for DS-CDMA using Sectoring for $\sigma_{d B}=7$ with an SNR per Bit of $15 \mathrm{~dB} \quad\left(\mathrm{R}_{\mathrm{cc}}=1 / 2\right.$ and $\left.\mathrm{v}=8\right)$.

intended mobile in the cell is represented by a random variable, which we incorporate into a revised probability of bit error.

To simplify our analysis, we will replace our hexagonal cells with overlapping circular cells. The overlap accounts for soft-handoff of mobile users between cells. We take the center base station to be the origin of a polar coordinate system. Accordingly, the position of our intended mobile user in the center cell is represented by $(r, \theta)$, where $0<\mathrm{r} \leq 1$ and $-\pi<\theta \leq \pi$. The randomization of the distances complicates the use of the Hata model in predicting path loss. Accordingly, the performance analysis in this section will use the average path loss $\overline{L_{\mathrm{n}}}(d) \propto d^{\mathrm{n}}$, with a path loss exponent of $n=4$.

In order to incorporate user distribution into our performance analysis of the forward channel, we require a joint probability density function in terms of $r$ and $\theta$ that represents a user's position within the cell. We define a user density as the normalized number of users per unit of area at a distance $r$ from the base station [11]. By assuming that the user density is uniform in [10], we developed the joint pdf for a user's position in the cell given by

$$
\mathrm{p}_{\mathrm{R}, \Theta}(\mathrm{r}, \theta)=\frac{\mathrm{r}}{\pi}, \quad 0<\mathrm{r} \leq 1, \quad-\pi<\theta \leq \pi
$$

We modify our expression for the first-event error probability defined by (9) (conditioned on $\mathrm{R}=\mathrm{r}$ and $\Theta=\theta$ ) as follows:

$$
\tilde{\mathrm{P}_{2}}(d)=\int_{-\pi}^{\pi} \int_{0}^{1} \int_{-\infty}^{\infty} \mathrm{Q}\left(\sqrt{\frac{z_{d}}{\hat{\alpha}}}\right) \mathrm{p}_{z_{d}}\left(z_{d}\right) \mathrm{p}_{\mathrm{R}, \Theta}(\mathrm{r}, \theta) d z_{d} d \mathrm{r} d \theta
$$

where

$$
\hat{\alpha}=\frac{\mathrm{E}\{X\}}{3 N} \sum_{i=1}^{6} \sum_{j=0}^{K_{i}-1} \frac{\mathrm{F}_{i j}}{\mathrm{~F}_{1}} \frac{\mathrm{r}^{\mathrm{n}}}{\mathrm{D}_{i}^{\mathrm{n}}}+\frac{\mathrm{N}_{0} \mathrm{r}^{\mathrm{n}}}{2 E_{c} \mathrm{r}_{\mathrm{f}}^{\mathrm{n}}} .
$$

The distances from the mobile to each of the adjacent base stations is represented in $D_{i}(r, \theta)$ as developed in [10]. The upper bound on the probability of bit error is given by (10) 
using the first-event error probability as defined in (12) and (13). Furthermore, we can implement antenna sectoring, which simply reduces the number of sectored users in cell $i$ to $K_{i} / S$, where $S$ is the number of sectors. We simulated the integral in (12) for the sectored system using 100,000 Monte Carlo trials and found that accommodating the linear user distribution into the analysis resulted in performance that was 3 to $4 \mathrm{~dB}$ better than that predicted by our worst-case scenario.

\section{B. Implementing Power Control}

The goal in our power-controlled system is to ensure that the power received by all mobile users is at the target power level $\mathrm{P}$ by adjusting the transmit power in each channel, $k$, with the power control factor $\mathrm{F}_{k}$. We can relate the target power received to the baseline transmit power $\mathrm{P}_{t}$ by an attenuation factor as $\mathrm{P}=\mathrm{P}_{\mathrm{t}} / \mathrm{a}$, where $\mathrm{P}, \mathrm{P}_{\mathrm{t}}$, and a are constant for all users in all cells. When the mobile user receives the information signal, the actual power received is measured and reported back to the base station for adjustment. At the point the mobile user measures his received power and reports it to the base station, it is no longer a random variable as described by (3), rather it is a realization of the random variable, which is known precisely and represented in our analysis as follows:

$$
\mathrm{P}_{k}=\frac{\mathrm{P}_{\mathrm{t}, k}}{\overline{L_{\mathrm{n}}}\left(d_{k}\right) x_{k}}=\frac{\mathrm{F}_{k} \mathrm{P}_{\mathrm{t}}}{\overline{L_{\mathrm{n}}}\left(d_{k}\right) x_{k}}=\frac{\mathrm{F}_{k} \mathrm{aP}}{d_{k}^{\mathrm{n}} x_{k}},
$$

where $d_{k}$ is the user's actual distance to the base station, and $x_{k}$ is the shadowing experienced by the user. Accordingly, in order for the power received by all users to be at the target level $\mathrm{P}$ the base station must adjust the power factor such that

$$
\mathrm{F}_{k}=\frac{d_{k}^{\mathrm{n}} x_{k}}{\mathrm{a}}
$$

With perfect power control, the power received by intended mobile is simply $\mathrm{P}$. Accordingly, by adjusting the power factor to achieve the target power received by all users, we have defeated the lognormal shadowing effect on the information signal. The power received from the adjacent cells, however, is a random variable based upon user distribution and lognormal shadowing. The first-event error probability for the power-controlled system is given by

$$
\widetilde{\mathrm{P}_{2}}(d)=\frac{1}{\pi} \int_{-\pi}^{\pi} \int_{0}^{1} \mathrm{r} \mu^{d} \sum_{k=0}^{d-1}\left(\begin{array}{c}
d-1+k \\
k
\end{array}\right)(1-\mu)^{k} d \mathrm{r} d \theta,
$$

where,

$$
\mu=\frac{1}{2}\left(1-\sqrt{\frac{2(\mathrm{E}\{X\})^{2}}{3 N} \sum_{i=1}^{6} \sum_{j=0}^{K_{i}-1} \frac{\mathrm{E}\left\{\mathrm{r}_{i j}^{\mathrm{n}}\right\}}{\mathrm{D}_{i}^{\mathrm{n}}}+\frac{\mathrm{N}_{0}}{E_{c}}+1}\right) .
$$

We can calculate an upper bound on the probability of bit error numerically using (16) and (17) in (10). Fig. 4 compares the probability of bit error for a cellular system using forward power control with the fixed-power results from Section IV.A.

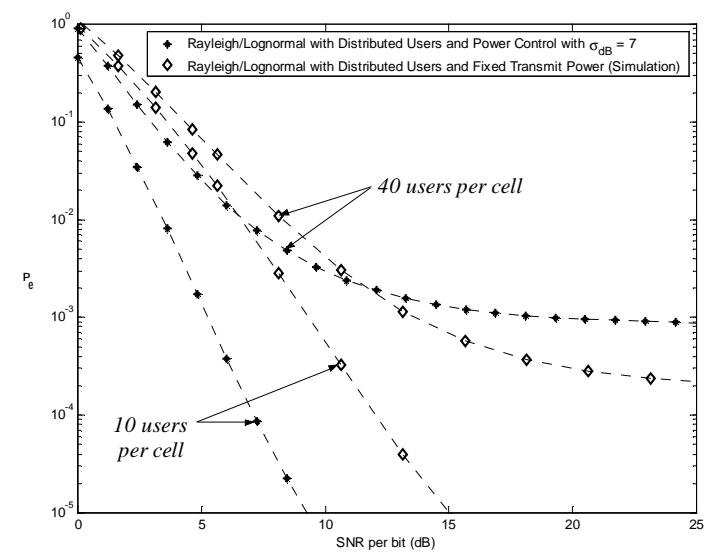

Fig. 4 Probability of Bit Error for DS-CDMA with Rayleigh Fading and Lognormal Shadowing $\left(\sigma_{d B}=7\right)$ using Forward Power Control and Linear User Distribution with $120^{\circ}$ Sectoring and FEC $\left(\mathrm{R}_{\mathrm{cc}}=1 / 2\right.$ and $\left.v=8\right)$.

In the case of 10 users per cell, the power-controlled system clearly outperforms the system with fixed power $(\sim 5 \mathrm{~dB})$. For 40 users per cell, however, the powercontrolled system outperforms the fixed-power system for $\overline{\gamma_{b}}<12 \mathrm{~dB}$, at which point the interference floor begins to develop. We see that the interference floor for the powercontrolled system is actually higher than the interference floor for the fixed-power system. In order to explain this, let us examine the isolated co-channel interference contribution from (17) as it compares with its equivalent expression in (13) for the fixed-power system. In order to ensure that the received power for all users is equal in the power-controlled system, we adjusted the power in the forward channel to overcome lognormal shadowing effects. By doing so, we have in effect increased the expected value of the co-channel interference by a factor of $E\{X\}=\exp \left(\lambda^{2} \sigma_{d B}^{2} / 2\right)$ over the fixed-power system. This extra factor of $E\{X\}$ is a result of randomizing the transmit power in the forward channel.

In spite of the increased co-channel interference, the performance of the power-controlled system is far superior to that of the fixed-power system for lognormal shadowing environments of $\sigma_{d B}<6$ with 120 degree antenna sectoring as shown in [10]. Under these circumstances, the benefits eliminating the lognormal shadowing from the intended signal outweigh the increased co-channel interference. Consequently, we can achieve a probability of bit error of less than $10^{-3}$ without entering the interference-limited region of operation. As shown in [10], the power-controlled system can accommodate as many as 100 users per cell using 120 degree sectoring for $\sigma_{d B}<6$. For $\sigma_{d B}=6$, the power- 
controlled system can accommodate up to 80 users using 120 degree sectoring and still achieve the desired probability of bit error of $10^{-3}$.

In designing a system with power control that operates in more intense lognormal shadowing environments $\left(\sigma_{d B}<6\right)$, we must ensure that our system is not working in the interference-limited region. For example, if we expect our system to work in the $\overline{\gamma_{b}}=15 \mathrm{~dB}$ range, the system could accommodate up to roughly 30 users per cell in a $\sigma_{d B}=7$ shadowing environment using 120 degree sectoring. Adding more users would degrade performance due to operating in the interference-limited region as indicated in Fig.5.

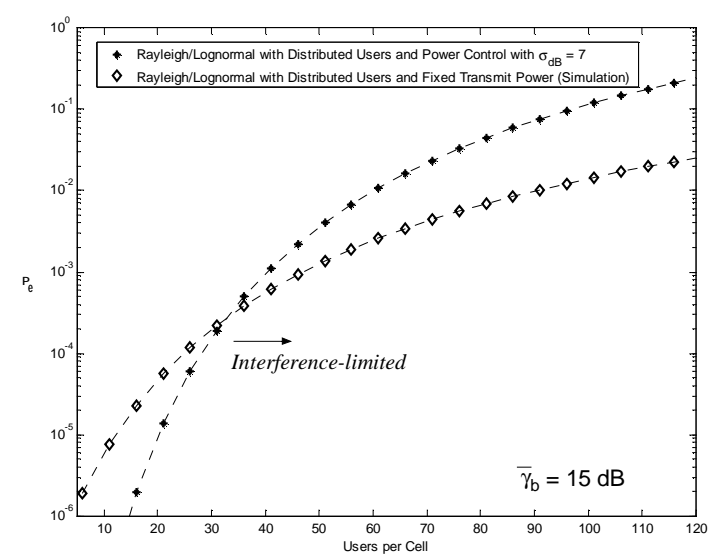

Fig. 5 Probability of Bit Error for DS-CDMA with Rayleigh Fading and Lognormal Shadowing $\left(\sigma_{d B}=7\right)$ vs. Users per Cell using Linear User Distribution with $120^{\circ}$ Sectoring and FEC $\left(\mathrm{R}_{\mathrm{cc}}=1 / 2\right.$ and $\left.v=8\right)$.

If a greater user capacity is required, we could increase the number of sectors per cell to six. The power-controlled system operating in a $\sigma_{d B}=7$ shadowing environment could support up to 70 users per cell using 60 degree antenna sectoring without operating in the interference-limited region.

The use of sectoring to alleviate the co-channel interference in support of power control only works up to a point. For a shadowing environment of $\sigma_{d B}=8$, for example, the 6-sector system can accommodate up to roughly 27 users prior to becoming interference-limited at an SNR per bit of $\gamma_{b}=15 \mathrm{~dB}$. In an environment where $\sigma_{d B}=9$, however, even with 60 degree sectoring we are interference limited with as few as 12 users working at $\bar{\gamma}_{b}=15$. Accordingly, implementing power control in such environments demands some additional method of interference mitigation for the system to be viable. Complete performance results for the power-controlled system using 60 degree sectoring can be found in [10].

\section{CONCLUSIONS}

In this paper we presented the performance analysis of the DS-CDMA forward channel operating in a Rayleigh-faded and lognormal-shadowed environment. We have incorporated forward error correction in the form of convolutional encoding with soft-decision decoding. We examined the performance of the system for the worst-case scenario with the mobile users at the edge of the cell, and accounting for a distributed user scenario. We added powercontrol to the forward channel, and found that performance and system capacity are greatly enhanced with careful implementation of power control.

\section{REFERENCES}

[1] J. G. Proakis, Digital Communications, Boston, Massachusetts. WCB/McGraw-Hill, 1993.

[2] T. S. Rappaport, Wireless Communications: Principles \& Practice, Upper Saddle River, New Jersey: Prentice Hall PTR, 1996.

[3] F. Adachi, M. Sawahashi, H. Suda, "Wideband DS-CDMA for NextGeneration Mobile Communications Systems," IEEE Commun. Magazine, pp. 56-69, Sep. 1988.

[4] O. K. Tonguz, M. M. Wang, "Cellular CDMA Networks Impaired by Rayleigh Fading: System Performance with Power Control," IEEE Trans. on Vehic. Tech., vol. 43, no. 3, pp. 515-527, Aug. 1994.

[5] L. B. Milstein, T. S. Rappaport, R. Barghouti, "Performance Evaluation for Cellular CDMA," IEEE Journ. Select. Areas. Commun., vol. 10, no. 4, pp. 680-689, May 1992.

[6] W. Ye and A. M. Haimovich, "Performance of Cellular CDMA with Cell Site Antenna Arrays, Rayleigh Fading, and Power Control Error," IEEE Trans. on Commun., vol. 48, no. 7, pp. 1151-1159, July 2000.

[7] T. T. Tjhung and C. C. Chai, "Distribution of SIR and Performance of DS-CDMA Systems in Lognormally Shadowed Rician Channels," IEEE Trans. on Vehic. Tech., vol. 49, no. 4, pp. 1110-1125, July 2000.

[8] B. Hashem and E. S. Sousa, "On the Capacity of Cellular DS/CDMA Systems Under Slow Rician/Rayleigh-Fading Channels," IEEE Tran. on Vehic. Tech., vol. 49, no. 5, pp. 1752-1759, Sep. 2000.

[9] COST 231, "Urban Transmission Loss Models for Mobile Radio in the 900 and 1,800 MHz bands (Revision 2)," COST $231 \mathrm{TD}(90) 119$ Rev. 2, The Hague, The Netherlands, Sep. 1991.

[10] J. E. Tighe, 'Modeling and Analysis of Cellular CDMA Forward Channel," Ph.D. Dissertation, Department of Electrical and Computer Engineering, Naval Post Graduate School, Monterey, CA, Mar. 2001.

[11] N. Abramson, "The Throughput of Packet Broadcasting Channels," IEEE Trans. on Commun. vol. COM-25, pp. 117-128, Jan. 1977. 